\title{
Accuracy of a Novel Non-Invasive technology based EZSCAN system for the diagnosis of diabetes mellitus in Chinese
}

\author{
Chang-Sheng Sheng ${ }^{1}$, Wei-Fang Zeng ${ }^{1}$, Qi-Fang Huang ${ }^{1}$, Jean-Paul Deslypere ${ }^{2}$, Yan Li ${ }^{1}$ and Ji-Guang Wang ${ }^{1 *}$
}

\begin{abstract}
Background: A new simple technique based on iontophoresis technology (EZSCAN, Impeto Medical, Paris, France) has recently been developed for the screening of diabetes. In the present study, we investigated the accuracy of this system for the diagnosis of diabetes mellitus in Chinese.

Methods: We performed the EZSCAN test in diabetic and non-diabetic subjects. EZSCAN measures electrochemical conductance (EC) at forehead, hands and feet, and derives a diabetes index with a value ranging from 0 to 100. Diabetes mellitus was defined as a plasma glucose concentration of at least $7 \mathrm{mmol} / \mathrm{l}$ at fasting or $11.1 \mathrm{mmol} / \mathrm{l}$ at 2 hours after glucose load, or as the use of antidiabetic drugs.

Results: The 195 study participants (51\% men, mean age 52 years) included 75 diabetic patients (use of antidiabetic drugs $81 \%)$ and 120 non-diabetic subjects. EC (micro Siemens, $\mu$ Si) was significantly $(P<0.001)$ lower in diabetic patients at the hands ( 44 vs. 61) and feet ( 51 vs. 69) locations, but not at the forehead (15 vs. $17, P=$ 0.39). When a diabetes index of 40 (suggested by the manufacturer) was used as the threshold, the sensitivity and specificity for the diagnosis of diabetes mellitus was $85 \%$ and $64 \%$, respectively. In 80 patients who underwent an oral glucose tolerance test, EC at hands and feet and the diabetes index were significantly $(P<0.001)$ associated with both 2-hour post-load plasma glucose and serum glycosylated haemoglobin.
\end{abstract}

Conclusions: EZSCAN might be useful in screening diabetes mellitus with reasonable sensitivity and specificity.

Keywords: EZSCAN, Diabetes mellitus, lontophoresis technology

\section{Background}

The prevalence of type 2 diabetes mellitus is high in most countries. According to the most recent nationwide survey in China, the prevalence of diabetes mellitus and impaired glucose metabolism was $9.7 \%$ and $15.5 \%$, respectively [1]. While diabetes mellitus is becoming a major public health problem, the identification of this disease is still difficult [2]. The oral glucose tolerance test (OGTT) is complicated. The measurement of glycosylated haemoglobin (HbA1c) requires high cost, and has low sensitivity, and no fully standardized analytical method. The fasting blood glucose measurement either

\footnotetext{
* Correspondence: jiguangw@gmail.com

${ }^{1}$ Centre for Epidemiological Studies and Clinical Trials, The Shanghai Institute of Hypertension, Ruijin Hospital, Shanghai Jiaotong University School of Medicine, Shanghai, China

Full list of author information is available at the end of the article
}

via venous or capillary sampling is currently used for screening, but is not sufficiently sensitive. Indeed, if fasting blood glucose was used solely in the diagnosis of diabetes mellitus, the sensitivity was as low as 40\% [3].

The recently developed EZSCAN system employs a novel technology of reverse iontophoresis [4] and measures function of sweat glands as a parameter of small nerve fiber dysfunction that may present early in prediabetes $[5,6]$. The original measurement of the system is sweat chloride concentration, while administering a lowvoltage electric current at the feet, hands and forehead. With an algorithm accounting for sex, age, body mass index, and systolic blood pressure, an index could be derived for the diagnosis of diabetes mellitus, impaired glucose metabolism, and diabetic neuropathy. The system has been tested in Europe [7] and India [8] for the diagnostic accuracy of diabetes mellitus. In the present 
Chinese study, we first investigated the accuracy of the derived diabetes index in the diagnosis of diabetes mellitus, and then correlated this index and the original electrochemical conductance measurement with concentrations of 2-hour post-load plasma glucose and serum HbA1c.

\section{Methods}

\section{Study subjects}

The study participants $(\mathrm{n}=195)$ were recruited from known diabetic patients enrolled in a trial of self blood glucose monitoring $(\mathrm{n}=54)$, from hypertensive patients hospitalized in the Department of Hypertension, Ruijin Hospital (Shanghai, China, $\mathrm{n}=96$ ), and from apparently healthy volunteers $(n=45)$. We performed the EZSCAN test in all these participants. In the 96 hospitalized hypertensive patients, we also performed OGTT and measured serum $\mathrm{HbA1c}$ concentration in those who had no history of diabetes mellitus $(\mathrm{n}=80)$ after exclusion of 12 patients with known diabetes mellitus and 4 patients who declined to participate. The Ethnics Committee of Ruijin Hospital, Shanghai Jiaotong University School of Medicine (Shanghai, China) approved the study protocol. All subjects gave written informed consent.

\section{The EZSCAN test}

The EZSCAN system is designed to evaluate sweat-gland function by measuring sweat chloride concentrations using reverse iontophoresis and chronoamperometry. Two sets of large-area nickel electrodes are used as anode and cathode. A direct current at an incremental voltage of $4 \mathrm{~V}$ or less is applied to the anode. This applied current generates voltage to the cathode and a current between the anode and cathode. This generated current is proportional to chloride concentration and measurable by chronoamperometry. The electrochemical conductance $(\mu \mathrm{Si})$ is calculated as the ratio of the current measured over the constant power applied for the forehead (left and right parts), hands (left and right), and feet (left and right) and for the whole body. A diabetes index is then derived from these electrochemical conductance measurements with an algorithm accounting for sex, age, body mass index, and systolic blood pressure, for the classification of glucose metabolism as the presence (diabetes index $\geq 40$ ) or absence of diabetes mellitus (diabetes index $<40$ ).

A trained physician (CSS) performed the EZSCAN test, after he administered a questionnaire to collect information on medical history and the use of medications, and measured body weight and body height. Blood pressure was measured three times consecutively after the subjects had rested for at least 5 minutes in the sitting position. These three blood pressure readings were averaged for analysis. Before the EZSCAN test, the operator had to enter the following variables into the system: sex, age, body height, body weight, and systolic blood pressure, but the subject did not require for any preparation, such as fasting or no vigorous exercise. During the 2-min test, the subject was asked to stand up still with the hands and feet on the pads of electrode and with the headband on the forehead.

\section{OGTT and laboratory methods}

A standard OGTT was performed after overnight fasting of 8-10 $\mathrm{h}$ according to the WHO recommendations [9] in subjects without known diabetes mellitus. Blood samples were drawn immediately and 30,60, 120, and 180 minutes after an oral load of $75 \mathrm{~g}$ anhydrous glucose. Plasma glucose was measured by the glucose oxidase peroxidase method. Diabetes mellitus was defined as a plasma glucose of at least $7.0 \mathrm{mmol} / \mathrm{L}$ fasting or 11.1 $\mathrm{mmol} / \mathrm{L}$ at 2 hours after glucose load, or as the use of antidiabetic agents [9]. HbA1c was measured by the HPLC method [10].

\section{Statistical methods}

For database management and statistical analysis, we used SAS software (version 9.13, SAS Institute, Cary, NC, USA). Means and proportions were compared with the Student's $t$-test and Fisher's exact test, respectively. The Receiver Operating Characteristic (ROC) [11] curve was used to show sensitivity and specificity of the EZSCAN diabetes index for the diagnosis of diabetes mellitus. We calculated Pearson correlation coefficients to test the correlations of the original electrochemical conductance measurements and the derived diabetes index with concentrations of 2-hour post-load plasma glucose and serum HbA1c.

\section{Results}

195 participants included 99 (51\%) men and 75 (39\%) diabetic patients, of whom 61 (81\%) took antidiabetic medication. Diabetic patients, compared with non-diabetic subjects, were older $(+14$ years, $P<0.0001)$, and had a greater body mass index $\left(+2 \mathrm{~kg} / \mathrm{m}^{2}, P=0.0001\right)$ and higher systolic blood pressure $(+9 \mathrm{mmHg}, P=$ $0.003)$ and fasting plasma glucose $(+2 \mathrm{mmol} / \mathrm{L}$, Table 1$)$.

Diabetic patients, compared with non-diabetic subjects, had significantly $(P<0.0001)$ lower electrochemical conductance for the hands $(44 \mathrm{vs} .61 \mu \mathrm{Si})$, feet (51 vs. $69 \mu \mathrm{Si}$ ), and the whole body (37 vs. $49 \mu \mathrm{Si}$ ) but not for the forehead (15 vs. $17 \mu \mathrm{Si}, P=0.39$ ), and had significantly $(P<0.0001)$ higher EZSCAN diabetes index $(67$ vs. 35 , Table 2).

The ROC curve shows the diagnostic accuracy of the derived EZSCAN diabetes index for the diagnosis of diabetes mellitus (Figure 1). The area under the curve (AUC) was $80 \%$ of the total square. If the EZSCAN diabetes index of 40 was used as the threshold for the diagnosis of diabetes mellitus in all subjects, the sensitivity and specificity 
Table 1 Characteristics of the study participants

\begin{tabular}{lccc}
\hline Characteristic & $\begin{array}{c}\text { Diabetic patients* } \\
(\mathbf{n}=\mathbf{7 5})\end{array}$ & $\begin{array}{c}\text { Non-diabetic subjects } \\
(\mathbf{n}=\mathbf{1 2 0})\end{array}$ & $\boldsymbol{P}$ value \\
\hline Men, $\mathrm{n}(\%)$ & $34(45.3)$ & $65(54.2)$ & 0.23 \\
Age, years & $60.5 \pm 8.4$ & $47.0 \pm 16.2$ & 0.0001 \\
Body height, $\mathrm{cm}$ & $162.7 \pm 9.3$ & $166.4 \pm 9.1$ & 0.006 \\
Body weight, $\mathrm{kg}$ & $70.4 \pm 12.7$ & $68.3 \pm 12.9$ & 0.26 \\
Body mass index, $\mathrm{kg} / \mathrm{m}^{2}$ & $26.6 \pm 3.9$ & $24.5 \pm 3.3$ & 0.0001 \\
Systolic blood pressure, $\mathrm{mm} \mathrm{Hg}$ & $143.5 \pm 14.2$ & $136.4 \pm 18.5$ & 0.003 \\
Diastolic blood pressure, $\mathrm{mm} \mathrm{Hg}$ & $80.2 \pm 9.2$ & $81.8 \pm 10.2$ & 0.50 \\
Fasting blood glucose, $\mathrm{mmol} / \mathrm{L}$ & $6.86 \pm 1.23$ & $5.07 \pm 0.59$ & $<.0001$ \\
Use of anti-diabetic drugs, $\mathrm{n} \mathrm{( \% )}$ & $61(81.3 \%)$ & 0 & - \\
\hline
\end{tabular}

Values are mean \pm standard deviation, or number of subjects (\%).

*Diabetes mellitus was defined as a plasma glucose of at least $7.0 \mathrm{mmol} / \mathrm{L}$ fasting or $11.1 \mathrm{mmol} / \mathrm{L}$ at 2 hours post-load, or as the use of antidiabetic agents.

were $85 \%$ and $64 \%$, respectively. If the threshold increased to 50 and 60 , the diagnostic sensitivity dropped to $67 \%$ and $60 \%$, respectively, and the diagnostic specificity rose to $72 \%$ and $78 \%$, respectively (Table 3 ). In 80 patients who had no history of diabetes mellitus and had an OGTT, 9 (11.3\%) were diagnosed as diabetes mellitus by OGTT. In these patients, the EZSCAN diabetes index of 40 had higher sensitivity (78\%) than fasting plasma glucose $(44 \%)$ or serum HbA1c (56\%, Table 3).

After stratification for sex and age (below or above the median of 54 years), we calculated sensitivity and specificity of the diabetes index of 40 in men $(n=99)$ and women $(\mathrm{n}=96)$ and in the younger $(\mathrm{n}=100)$ and older $(\mathrm{n}=95)$ subjects. The EZSCAN system had similar sensitivity and specificity in both sexes $(P \geq 0.50)$, but tended to have higher sensitivity and lower specificity in older than younger subjects $(P \leq 0.003$, Table 3$)$.

In further analysis, we correlated the original electrochemical conductance measurement and the derived EZSCAN diabetes index with concentrations of 2-hour postload plasma glucose and serum HbA1c in 80 patients who had an OGTT. The electrochemical conductance was significantly $(P \leq 0.009)$ and negatively correlated with both 2 hour post-load plasma glucose concentration and serum HbA1c at the hands $(r=-0.35$ and $r=-0.35$, respectively) and feet $(\mathrm{r}=-0.29$ and $\mathrm{r}=-0.38$, respectively) but not $(P \geq$ $0.53)$ at the forehead $(r=-0.02$ and $r=0.07$, respectively, Figure 2). The EZSCAN diabetes index was significantly $(P \leq 0.004)$ correlated with 2-hour post-load glucose $(\mathrm{r}=$ $0.32)$ and $\operatorname{HbA} 1 \mathrm{C}(\mathrm{r}=0.38$, Figure 3$)$.

\section{Discussion}

Our study demonstrated that EZSCAN, as a screening tool, had an acceptable accuracy for the diagnosis of diabetes mellitus. Indeed, if a diabetes index of 40 would be used as the threshold, the diagnostic sensitivity and specificity was $85 \%$ and $64 \%$, respectively. In addition, the electrochemical conductance at the hands and feet but not at the forehead was significantly associated with 2hour post-load plasma glucose concentration and serum HbA1C concentration.

To the best of our knowledge, only 2 published studies investigated the accuracy of EZSCAN for the diagnosis of impaired glucose metabolism. In 212 subjects recruited in India [7], EZSCAN, when a diabetes index of 50 was used as the threshold, had a sensitivity of $75 \%$ for the diagnosis of diabetes mellitus $(n=24)$, and a specificity of $54 \%$ in patients with normal glucose tolerance and without the metabolic syndrome $(n=101)$. The corresponding sensitivity and specificity for fasting

Table 2 The electrochemical conductance and the EZSCAN diabetes index in diabetic and non-diabetic subjects

\begin{tabular}{|c|c|c|c|c|}
\hline & $\begin{array}{l}\text { Diabetic patients* } \\
\qquad(\mathrm{n}=75)\end{array}$ & $\begin{array}{l}\text { Non-diabetic subjects } \\
\qquad(\mathrm{n}=120)\end{array}$ & $\begin{array}{c}\text { Relative difference } \\
\text { (95\% confidence interval) }\end{array}$ & $P$ value \\
\hline \multicolumn{5}{|l|}{ Electrochemical conductance, $\mu \mathrm{Si}$} \\
\hline Feet & $50.9 \pm 21.9$ & $69.1 \pm 16.8$ & $-26.3 \%(-18.4-34.3 \%)$ & $<0.0001$ \\
\hline Hands & $43.7 \pm 20.4$ & $61.2 \pm 15.5$ & $-28.6 \%(-20.3-36.9 \%)$ & $<0.0001$ \\
\hline Forehead & $15.1 \pm 17.8$ & $17.4 \pm 18.8$ & $-13.2 \%(-44.3-17.8 \%)$ & 0.39 \\
\hline Overall & $36.6 \pm 15.8$ & $49.2 \pm 11.8$ & $-25.8 \%(-17.7-33.7 \%)$ & $<0.0001$ \\
\hline \multicolumn{5}{|l|}{ EZSCAN diabetes index (0-100) } \\
\hline Mean \pm SD & $66.6 \pm 25.8$ & $34.6 \pm 27.9$ & $92.5 \%(70.0-115.6 \%)$ & $<0.0001$ \\
\hline Median (inter-quartile range) & $68(48)$ & $29(47)$ & - & $<0.0001$ \\
\hline
\end{tabular}

Values are mean \pm standard deviation, unless otherwise indicated.

*Diabetes mellitus was defined as a plasma glucose of at least $7.0 \mathrm{mmol} / \mathrm{L}$ fasting or $11.1 \mathrm{mmol} / \mathrm{L}$ at 2-hour post-load, or as the use of antidiabetic agents. 


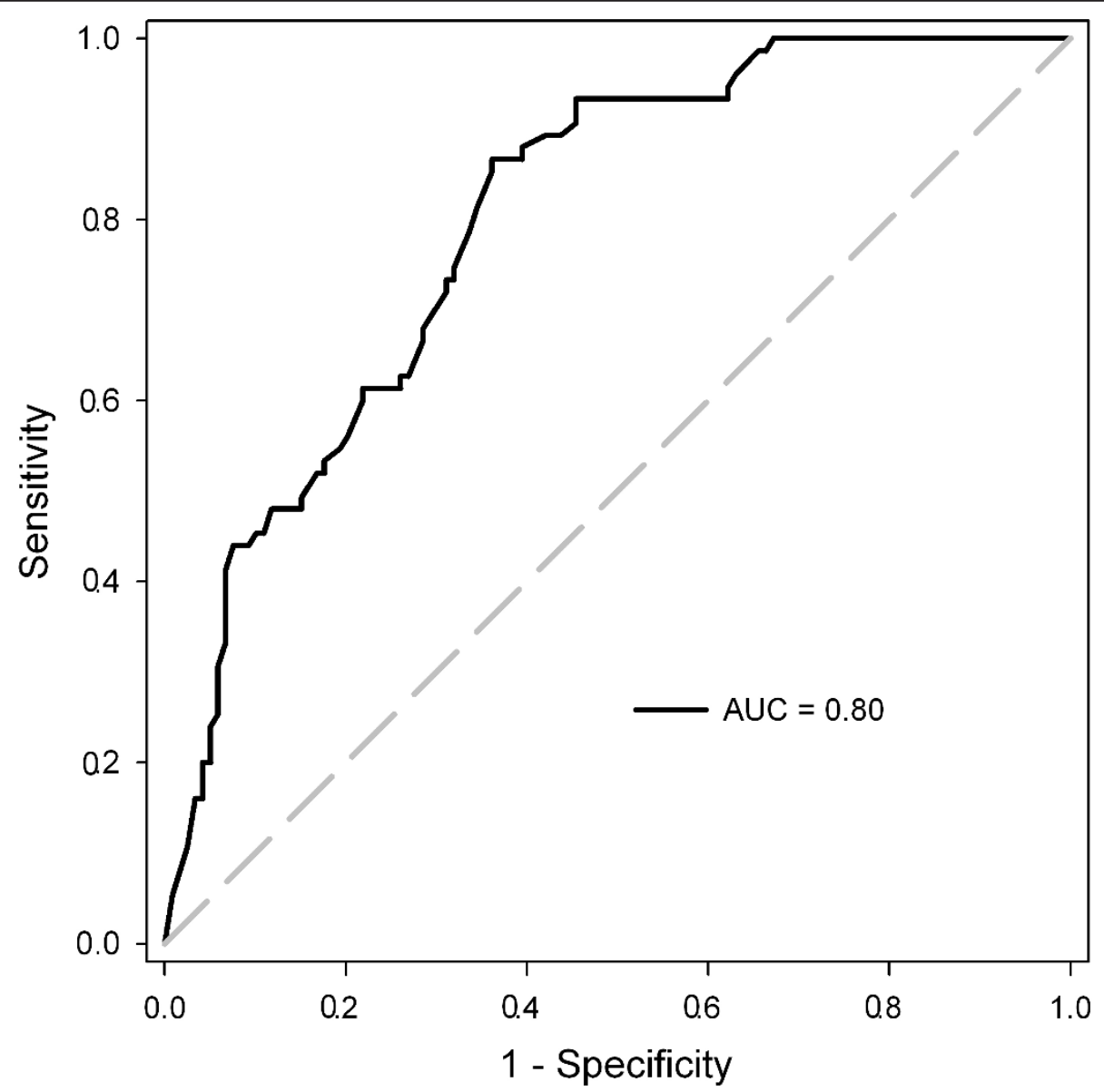

Figure 1 Accuracy of EZSCAN for the diagnosis of diabetes mellitus, analyzed by Receiver Operating Characteristic (ROC) curve.

plasma glucose ( $\geq 7.0 \mathrm{mmol} / \mathrm{L})$ were $29 \%$ and $99 \%$, respectively. In Indians, EZSCAN apparently had higher sensitivity but lower specificity than fasting glucose. In
133 diabetic patients and 41 healthy volunteers recruited in France [8], EZSCAN had a sensitivity of $75 \%$ and a specificity of $100 \%$.

Table 3 Sensitivity and specificity for the diagnosis of diabetes mellitus

\begin{tabular}{|c|c|c|}
\hline & Sensitivity (\%) & Specificity (\%) \\
\hline \multicolumn{3}{|l|}{ EZSCAN in all subjects $(n=195)^{*}$} \\
\hline EZSCAN diabetes index $\geq 40$ & 85 & 64 \\
\hline EZSCAN diabetes index $\geq 50$ & 67 & 72 \\
\hline EZSCAN diabetes index $\geq 60$ & 60 & 78 \\
\hline \multicolumn{3}{|c|}{ EZSCAN in subgroup analysis (diabetes index $\geq 40$ as threshold) } \\
\hline Men $(n=99)$ & 82 & 65 \\
\hline Women $(n=96)$ & 87 & 63 \\
\hline$<54$ years (median age, $\mathrm{n}=100$ ) & 61 & 83 \\
\hline$\geq 54$ years (median age, $n=95$ ) & 93 & 24 \\
\hline \multicolumn{3}{|c|}{ Comparison of 3 methods in patients who had an oral glucose tolerance test $(n=80)^{*}$} \\
\hline EZSCAN diabetes index $\geq 40$ & 78 & 67 \\
\hline Fasting plasma glucose $\geq 7.0 \mathrm{mmol} / \mathrm{L}$ & 44 & 100 \\
\hline Serum glycosylated haemoglobin $\geq 6.5 \%$ & 56 & 93 \\
\hline
\end{tabular}

*Diabetes mellitus was defined as a plasma glucose of at least $7.0 \mathrm{mmol} / \mathrm{L}$ fasting or $11.1 \mathrm{mmol} / \mathrm{L}$ at 2 hours post-load, or as the use of antidiabetic agents in all subjects (75 diabetic patients), and as a plasma glucose of at least $7.0 \mathrm{mmol} / \mathrm{L}$ fasting or $11.1 \mathrm{mmol} / \mathrm{L}$ at 2 hours post-load in patients who had an oral glucose tolerance test (9 diabetic patients). 


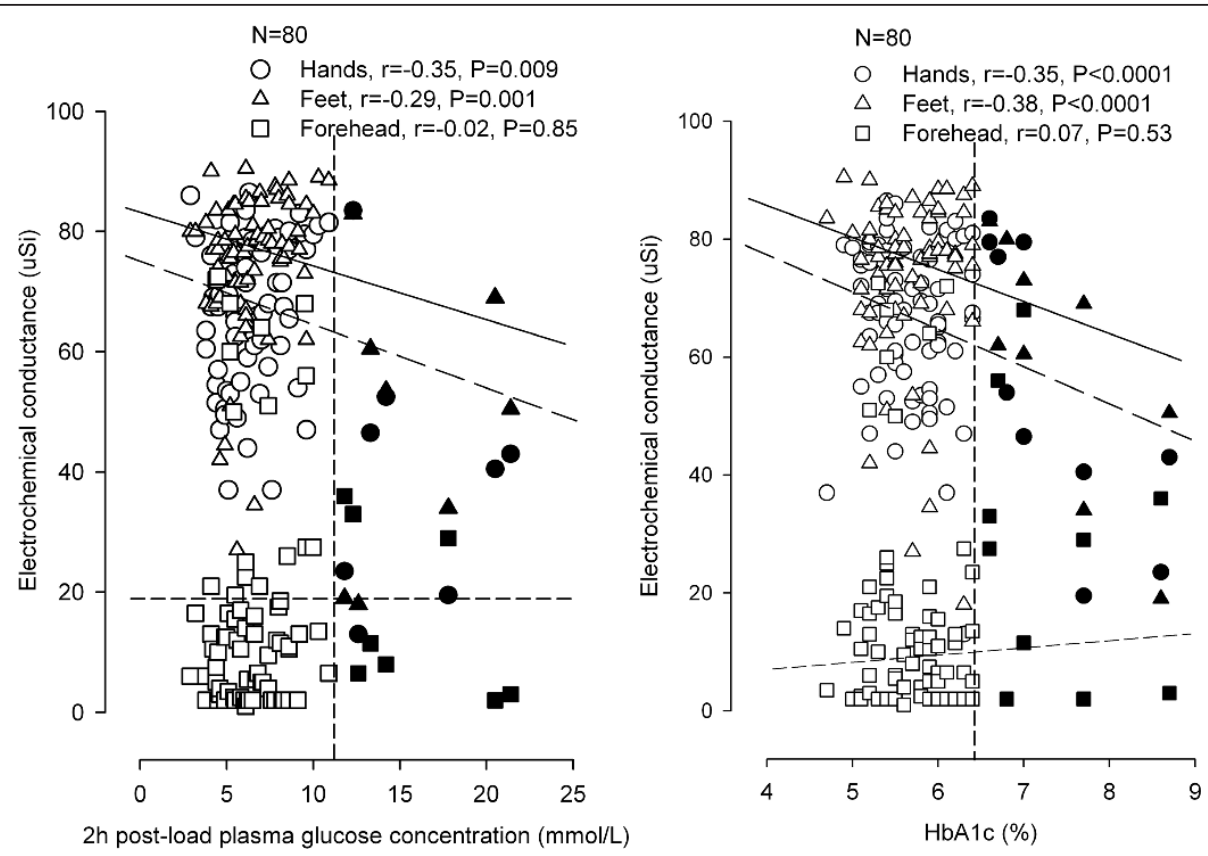

Figure 2 Scatter plots with regression line for the relation of the electrochemical conductance at the hands (circles), feet (triangles) and forehead (squares) locations with 2-hour post-load plasma glucose concentration (left panel) and serum glycosylated haemoglobin $(\mathrm{HbA1c}$, right panel) in patients who had an oral glucose tolerance test $(\mathbf{n}=\mathbf{8 0})$. The dashed vertical line denotes the threshold for the diagnosis of diabetes mellitus. Open and closed symbols represent diabetic patients and non-diabetic subjects, respectively. Correlation coefficients and $\mathrm{P}$ values are given for the feet, hands and forehead locations separately.

Taken the results of the 2 previous studies and our research together, EZSCAN seemed to have consistent and constant sensitivity $(75 \%$ in Indians and French and $85 \%$ in Chinese) but divergent and variable specificity
$(54 \%, 100 \%$, and $64 \%$, respectively) across populations. The heterogeneous specificity might be attributable to the differences in characteristics of participants between these 3 studies, because subjects who had any risk factor

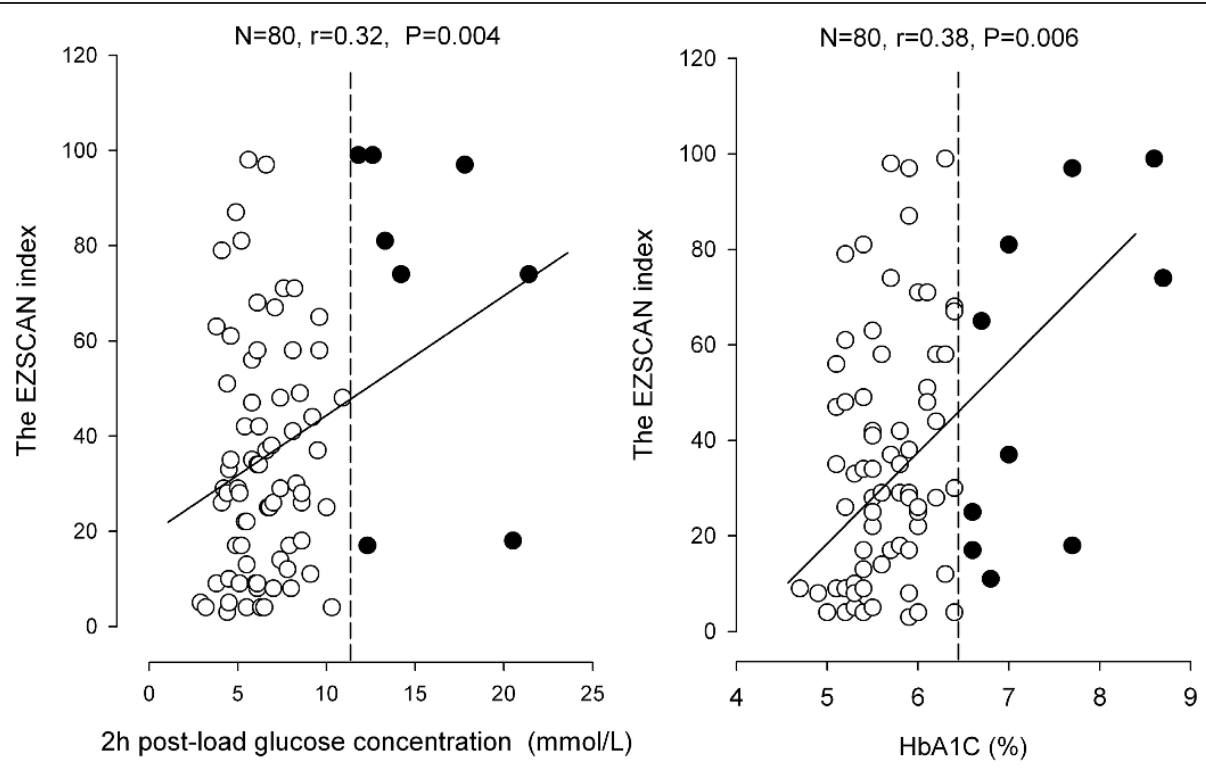

Figure 3 Scatter plots with regression line for the relation of the EZSCAN diabetes index with 2-hour post-load plasma glucose concentration (left panel) and serum glycosylated haemoglobin (HbA1c, right panel) in patients who had an oral glucose tolerance test $(\mathbf{n}=\mathbf{8 0})$. For further information, see legend to Figure 2 . 
for diabetes mellitus ( $\geq 45$ years, infrequent physical activity, and first-degree relative with diabetes) were excluded from the non-diabetic control group of the French study [8] but not the Indian [7] and our Chinese studies. Nonetheless, it is also possible that the algorithm for the computation of the diabetes index and the cutoff value for the diagnosis of diabetes mellitus generated from the French population cannot directly be generalized to populations of other ethnicities.

Despite different diagnostic accuracy across populations, EZSCAN does measure something that reflects or is related to glucose metabolism. The original electrochemical conductance measurement at hands and feet was different between diabetic and non-diabetic subjects, and associated with 2-hour post-load plasma glucose and with serum HbA1c. The EZSCAN diabetes index was also associated with 2-hour post load plasma glucose and serum HbA1c.

Our finding that the electrochemical conductance was significantly different between diabetic and non-diabetic subjects only at the feet and hands locations but not at forehead are in keeping with the results of the French study [8], and might have important implications for future upgrading of the EZSCAN system. In the French study, diabetic patients, compared with non-diabetic subjects, had a lower electrochemical conductance at the feet and hands locations but an even higher value at forehead [8]. The electrochemical conductance measures function of sweat glands and activity of the corresponding autonomic nerves that are longest at the feet, intermediate at the hands, and shortest at the forehead [12]. We speculate that longer nerves are more susceptible for neuropathic lesions, regardless as a complication or in reverse as a cause of diabetic mellitus [13-15]. Thus, only the electrochemical conductance at the feet and hands locations is sufficiently sensitive to identify the presence of diabetes mellitus, and should be used in the upgraded system.

One of the major strengths of our study was that we performed OGTT and measured HbA1c, and compared the accuracy of the EZSCAN system with that of fasting plasma glucose and HbA1c. Nonetheless, our study had a small sample size and a cross-sectional design. A larger prospective study is apparently required.

In conclusion, EZSCAN is accurate in the diagnosis of diabetes mellitus with reasonable sensitivity and specificity as a screening tool. With the increasing use and knowledge, EZSCAN might bring revolutionized changes to the identification of diabetes mellitus as a screening tool and also to the understanding of mechanisms of blood glucose regulation as a research tool. More research is apparently required to investigate the significance of EZSCAN for risk stratification and prediction of cardiovascular and renal events in prospective studies, and to explore the role of EZSCAN in therapeutic monitoring of diabetes mellitus in controlled clinical trials. Before the evidence is becoming available, this system should be recommended for the use in the research setting.

\section{Acknowledgements}

The authors gratefully acknowledge the voluntary participation of all study subjects. The study was financially supported by grants from the National Natural Science Foundation of China (30871360, 30871081 and 81170245), the Ministry of Science and Technology (2006BAl01A03 and a grant for China-European Union collaborations [1012]) and the Ministry of Education (NCET-09-0544), Beijing, China, the Shanghai Commission of Science and Technology (07JC14047 and the "Rising Star" program 06QA14043), the Shanghai Commission of Education (07ZZ32 and the "Dawn" project 08SG20), the Shanghai Bureau of Health (2009111 and XBR2011004), Shanghai Shenkang Hospital Development Centre (SHDC12007318), Shanghai Jiaotong University School of Medicine (a grant of Distinguished Young Investigators to Yan Li), and the European Union (LSHM-CT-2006037093 and HEALTH-F4-2007-201550).

\section{Author details}

'Centre for Epidemiological Studies and Clinical Trials, The Shanghai Institute of Hypertension, Ruijin Hospital, Shanghai Jiaotong University School of Medicine, Shanghai, China. ${ }^{2}$ Impeto Medical, Paris, France.

\section{Authors' contributions}

CSS carried out the field work, performed statistical analysis, and together with JGW drafted the manuscript. WFZ and QFH participated in the field work. JPD provided valuable help on the use of the EZSCAN system. YL and JGW conceived of, designed and coordinated the study. All authors read and approved the final manuscript.

\section{Competing interests}

The authors declare that they have no competing interests.

Received: 25 July 2011 Accepted: 22 December 2011

Published: 22 December 2011

\section{References}

1. Yang W, Lu J, Weng J, Jia W, Ji L, Xiao J, Shan Z, Liu J, Tian H, Ji Q, Zhu D, Ge J, Lin L, Chen L, Guo X, Zhao Z, Li Q, Zhou Z, Shan G, He J: China National Diabetes and Metabolic Disorders Study Group: Prevalence of diabetes among men and women in China. N Engl J Med 2010, 12:1090-101.

2. American Diabetes Association: Diagnosis and classification of diabetes mellitus. Diabetes Care 2011, 34:S62-9.

3. Engelgau MM, Narayan KM, Herman WH: Screening for type 2 diabetes. Diabetes Care 2000, 23:1563-80.

4. Delgado-Charro MB, Guy RH: Transdermal reverse iontophoresis of valproate: a non invasive method for therapeutic drug monitoring. Pharm Res 2003, 20:1508-13.

5. Vinik Al, Mason RE, Mitchell BD, Freeman R: Diabetic autonomic neuropathy. Diabetes Care 2003, 26:1553-79.

6. Hsu WC, Yen AM, Liou HH, Wang HC, Chen TH: Prevalence and risk factors of somatic and autonomic neuropathy in prediabetic and diabetic patients. J Neurol Neurosurg Psychiatry 1990, 53:558-63.

7. Mayaudon H, Miloche PO, Bauduceau B: A new simple method for assessing sudomotor function: relevance in type 2 diabetes. Diabetes Metab 2010, 36:450-4.

8. Ramachandran A, Moses A, Shetty S, Thirupurasundari CJ, Seeli AC, Snehalatha C, Singvi S, Deslypere JP: A new non-invasive technology to screen for dysglycaemia including diabetes. Diabetes Res Clin Pract 2010, 88:302-6.

9. Alberti KG, Zimmet PZ: Definition, diagnosis and classification of diabetes mellitus and its complications. Part 1: diagnosis and classification of diabetes mellitus provisional report of a WHO consultation. Diabet Med 1998, 15:539-53. 
10. Nasir NM, Thevarajah M, Yean CY: Hemoglobin variants detected by hemoglobin $\mathrm{A} 1 \mathrm{c}(\mathrm{HbA1c})$ analysis and the effects on $\mathrm{HbA1c}$ measurements. Int J Diabetes Dev Ctries 2010, 30:86-90.

11. Metz CE: Basic principles of ROC analysis. Semin NuCl Med 1978, 8:283-98.

12. Hoeldtke RD, Bryner KD, Horvath GG, Phares RW, Broy LF, Hobbs GR: Redistribution of sudomotor responses is an early sign of sympathetic dysfunction in type 1 diabetes. Diabetes 2001, 50:436-543.

13. Provitera V, Nolano M, Caporaso G, Stancanelli A, Santoro L, Kennedy WR: Evaluation of sudomotor function in diabetes using the dynamic sweat test. Neurology 2010, 74:50-6.

14. Lauria G, Devigili G: Skin biopsy as a diagnostic tool in peripheral neuropathy. Nat Clin Pract Neurol 2007, 3:546-57.

15. Umapathi T, Tan WL, Loke SC, Soon PC, Tavintharan S, Chan YH: Intraepidermal nerve fiber density as a marker of early diabetic neuropathy. Muscle Nerve 2007, 35:591-8.

doi:10.1186/1758-5996-3-36

Cite this article as: Sheng et al: Accuracy of a Novel Non-Invasive technology based EZSCAN system for the diagnosis of diabetes mellitus in Chinese. Diabetology \& Metabolic Syndrome 2011 3:36.

\section{Submit your next manuscript to BioMed Central} and take full advantage of:

- Convenient online submission

- Thorough peer review

- No space constraints or color figure charges

- Immediate publication on acceptance

- Inclusion in PubMed, CAS, Scopus and Google Scholar

- Research which is freely available for redistribution

Submit your manuscript at www.biomedcentral.com/submit 